\title{
Azul de Metileno na Paralisia Supranuclear Progressiva, uma possibilidade?
}

\author{
Methylene Blue in Progressive Supranuclear Palsy, one \\ option?
}

\section{Azul de metileno en la parálisis supranuclear progresiva, ¿una posibilidad?}

\begin{abstract}
Celmir de Oliveira Vilaça ${ }^{1,2}$, Bruno Lima Pessoa ${ }^{2}$, Marco Orsini ${ }^{3,4}$, Carlos Henrique Melo Reis ${ }^{3}$, Marco Antonio Alves Azizi ${ }^{3}$, Adalgiza Mafra Moreno ${ }^{3}$, Victor Hugo Bastos ${ }^{4}$, Marco Antônio Araujo Leite ${ }^{2}$

1.Instituto Nacional de Traumatologia e Ortopedia (INTO), Rio de Janeiro - RJ, Brasil.

2.Universidade Federal Fluminense. MMC/Divisão de Neurologia, HUAP. Programa de pós-graduação em Neurologia/Neurociências, UFF, Niterói-RJ, Brasil.

3.UNIG - Universidade Iguaçu e Hospital Geral da Posse, Nova Iguaçu, RJ, Brasil.

4.UFPI - Universidade Federal do Piaui - Departamento de Fisioterapia. Laboratório de Funcionalidade e Mapeamento Cerebral, Brasil.
\end{abstract}

\section{Resumo}

Objetivo. Discutir a hipótese do uso do azul de metileno na Paralisia Supranuclear Progressiva. Método. Revisão não sistemática empregando as palavras-chaves "methylene blue" associada aos termos "tauopathies, "tau protein", "Alzheimer's Disease", "Frontotemporal Dementia", "Progressive Supranuclear Palsy" e "Corticobasal Degeneration". Resultados. Foram utilizados 45 artigos, todos em inglês para a confecção deste artigo. Conclusão. $O$ uso de inibidores de agregação proteica como o azul de metileno representa importante área de pesquisa para tratamento das tauopatias. Trabalhos realizados com o uso do azul de metileno em tauopatias como a Doença de Alzheimer e a demência frontotemporal sugerem a possibilidade de seu uso na Paralisia Supranuclear Progressiva.

Unitermos. azul de metileno; tauopatias; paralisia supranuclear progressiva

\begin{abstract}
Objective. Make a discussion about hypothesis of the use of methylene blue in Progressive Supranuclear Palsy. Method. We perform a Non-systematic review using the words "methylene blue" associated with the terms "tauopathies", "tau protein", "Alzheimer's disease", "Frontotemporal dementia", "Progressive supranuclear palsy" and "Corticobasal" Degeneration. Results. We choose 45 papers, all in English language for of this article. Conclusion. Use of inhibitors of protein aggregation such as methylene blue represents an important area of research for the treatment of tauopathies. Tests with methylene blue in tauopathies such as Alzheimer's disease and frontotemporal dementia suggest a possibility of his utilization in Progressive Supranuclear Palsy.
\end{abstract}

Keywords. methylene blue; tauopathies; progressive supranuclear palsy

\section{Resumen}

Objetivo. Discuta la hipótesis del uso de azul de metileno en la parálisis supranuclear progresiva. Método. Revisión no sistemática con las palabras clave "azul de metileno" asociadas con los términos "tauopatías", proteína tau "," Enfermedad de Alzheimer "," Demencia frontotemporal "," Parálisis supranuclear progresiva "y" Degeneración corticobasal ". Resultados. Se utilizaron 45 artículos, todos en inglés para la elaboración de este artículo. Conclusión. El uso de inhibidores de la agregación de proteínas como el azul de metileno representa un área de investigación importante para el tratamiento de las tauopatías. Los estudios realizados con el uso de azul de metileno en tauopatías como la enfermedad de 
Palabras clave. azul de metileno; tauopatías; parálisis supranuclear progresiva

Trabalho realizado na Universidade Federal Fluminense - UFF, Niterói-RJ, Brasil.

\section{INTRODUÇÃO}

A paralisia Supranuclear progressiva (PSP) é considerada a segunda forma de parkinsonismo degenerativo mais frequente, correspondendo a $5 \%$ dos casos, atrás da Doença de Parkinson (DP) ${ }^{1}$. A PSP foi descrita inicialmente como síndrome em 1963 por John Steele, J. Clifford Richardson e Jerzy Olszewsly, também sendo denominada de Síndrome de Steele-Richardson-Olszwsky². Em 1964, estes mesmos autores realizaram análise de nove pacientes com PSP, através de estudo clínicos e neuropatológico ${ }^{1}$.

A PSP pertence à família das tauopatias. Estas doenças caracterizam-se por anormalidades na proteína tau associada a microtúbulos, causando degeneração em diferentes regiões cerebrais ${ }^{3}$. A prevalência de PSP varia entre 1,3 a 6,4 pessoas a cada 100 mil habitantes contra 4,4 da Atrofia de Múltiplos Sistemas (AMS), uma outra forma de Parkinsonismo atípico ${ }^{4,5}$. A doença apresenta pico de incidência aos 63 anos de idade, não sendo descrito casos 
em idade inferior aos 40 anos, com maior ocorrência em indivíduos do sexo masculino ${ }^{3,6}$.

Possui evolução progressiva e incurável, com sobrevida média de 9 anos e diagnóstico em média feito a partir de 4 anos do início dos sintomas com acometimento cortical e subcortical $^{1,3}$. A PSP clinicamente manifesta-se por um parkinsonismo atípico com sintomas motores (anormalidades do olhar voluntário, acinesia, rigidez, instabilidade postural, disartria e disfagia) associados a mudanças de personalidade e prejuízo cognitivo ${ }^{7}$. Atualmente além da forma clássica são reconhecidos diversas variantes clínicas da PSP. As demais formas de PSP apresentam em média maior tempo até o diagnóstico quando em comparação a forma clássica provavelmente pela ausência de oftalmoplegia até estágios mais avançados ${ }^{1,8}$.

Por outro lado, o azul de metileno (AzM) é o primeiro composto líquido sintético usado como antisséptico e primeiro a ser usado de forma terapêutica. Foi sintetizado pela primeira vez em 1876, por Heinrich Caro da Badische Anilin \& Soda Fabrik (BASF) como um líquido para tingir tecidos $^{9}$. Corresponde a um líquido catiônico de nome químico cloreto de metiltionínio, pertencentes a classe das fenotizianas. Durante a era pré-antibiótica foi largamente prescrito antes do advento da penicilina e das sulfonamidas como agente antibacteriano ${ }^{10}$.

Atualmente o AzM é usado em medicina como tratamento da metahemoglobinemia, encefalopatia induzida pela quimioterapia com ifosfamida e cirurgia tireoidiana. 
Pertencendo a família das fenotiazinas também apresenta efeitos em doenças psiquiátricas como psicose e mania ${ }^{11}$. Também pode ser usado na prevenção de infecções urinárias e visualização de fístulas assim como laboratorialmente é componente da coloração Giemsa, usada na visualização de parasitas em exames laboratoriais, como na malária ${ }^{12}$.

Mais recentemente descobriu-se o papel do AzM como inibidor da agregação proteica da proteína amiloide e da proteína tau assim como sua capacidade antioxidante, sendo utilizado em estudos de doenças neurodegenerativas como a Doença de Alzheimer ${ }^{12}$.

O objetivo do presente artigo é discutir a hipótese do uso do azul de metileno na PSP a partir dos periódicos, abordando sua eficácia em patologias caracterizadas pelo acúmulo de proteína tau.

\section{MÉTODO}

Foi utilizado para a confecção deste artigo a plataforma Google Scholar com seleção de artigos entre janeiro de 1992 a fevereiro de 2018. Empregou-se para a busca as palavraschave: "methylene blue" associada aos seguintes termos: tauopathies, "tau protein", "Alzheimer's Disease", "Frontotemporal Dementia", "Progressive Supranuclear Palsy" e "Corticobasal Degeneration" no título do periódico. Foram obtidos 34 artigos. Outros artigos foram utilizados a partir das referências dos trabalhos obtidos pela busca inicial. Utilizamos artigos originais, descritivos elou 
experimentais, artigos de revisão, opiniões de peritos e relatos de casos sem nenhum critério específico para escolha dos periódicos, exceto se encontrarem em língua inglesa. Ao todo utilizamos 45 artigos na confecção deste manuscrito.

\section{RESULTADOS e DISCUSSÃO}

\section{A Paralisia Suprenuclear Progressiva}

A PSP é definida neuropatologicamente pelo acúmulo de emaranhados neurofibrilares em diversas áreas do sistema nervoso central (SNC), como o núcleo subtalâmico, globo pálido, núcleo rubro, substância negra, tegmento pontino, núcleo oculomotor, bulbo e núcleo denteado ${ }^{1}$. É classificada como uma tauopatia pois na análise neuropatológica existe o achado de agregados protéicos consistindo de proteína tau (tubulin associated unit) ${ }^{3}$. A proteína tau foi descoberta em 1975 através de seu isolamento no tecido cerebral, recebendo sua designação pela letra grega tau $(T)$ devido sua capacidade de induzir a formação de túbulos através da montagem da tubulina. Considerada a mais abundante proteína ligada aos microtúbulos no cérebro é responsável pela manutenção do citoesqueleto axonal13,14.

O gene codificador da proteína tau está localizado no cromossomo $17 q 21$ possuindo 16 éxons no seu transcrito primário $^{3}$. Ele é conhecido como gene MAPT (Microtubule Associated Protein Tau). O locus do Gene MAPT existe como dois haplótipos $\mathrm{H} 1$ e $\mathrm{H} 2$ na população de origem europeia. A presença de haplótipo $\mathrm{H} 1 / \mathrm{H} 1$ é fator de risco maior para 
ocorrência de PSP. Ademais, mutações do gene MAPT podem causar PSP familiar de forma dominante embora a maioria dos casos sejam esporádicos ${ }^{15}$. A proteína tau apresenta 6 isoformas derivadas de 3 splicing alternativos dos éxons 2,3 e 10. A proteína tau apresenta domínios de ligação de microtúbulos contendo 3 ou 4 repetições de 31 aminoácidos (Formas 3R e 4R tau) ${ }^{5}$.

No cérebro fetal predomina a forma $3 R$ tau em relação a forma $4 R$ tau. Nos adultos a proporção encontrada das formas $3 R$ e $4 R$ tau são equivalentes, com proporção de 3:1 em favor da 4R tau em portadores de PSP pela inclusão do éxon 10 durante splicing ${ }^{5,16}$.

Além dos agregados de emaranhados neurofibrilares globoides, na neuropatologia da PSP há o achado de fios de neuropilos, astrócitos em tufos ou estrelados e corpos oligodendrogliais em molas ${ }^{1}$. Os astrócitos em tufo e fios de neuropilo são considerados os achados neuropatológicos mais específicos de PSP5 ${ }^{5}$. Esses agregados são formados por filamentos contínuos de proteína tau, diferentemente dos agregados pareados em hélice vistos na Doença de Alzheimer (DA), a mais frequente das tauopatias ${ }^{1}$.

Há também um grande percentual de indivíduos com PSP onde existe além da agregação tau achados neuropatológicos concorrentes e representativos de outras doenças degenerativas do SNC como os corpos argirofílicos da degeneração córtico basal, emaranhados e placas amiloides da DA e corpos de Lewy da DP ou Demência de 
Lewy ${ }^{4}$. É desconhecido se estes achados são responsáveis pelas diferentes formas clínicas de PSP ${ }^{17}$.

\section{Tratamento da PSP com drogas atuantes na proteína}

\section{Tau}

Agregados de proteína tau são encontrados em torno de 15 a 20 doenças degenerativas, algumas por mutação do gene tau em $\mathrm{si}^{18}$. Uma das prioridades nas pesquisas de tratamento das tauopatias está no desenvolvimento de compostos visando inibição da formação de agregados de proteína tau ${ }^{19}$. É sabido ser o desenvolvimento clínico de novos medicamentos de custo estimado no mínimo duas ordens de grandeza maior, quando em comparação as suas pesquisas de laboratório ${ }^{20}$.

Analisando-se as tauopatias, a neurodegeneração pode ocorrer pelo fato dos agregados tau serem tóxicos para as células. Sendo assim os tratamentos visando a inibição da agregação da proteína tau, redução da fosforilação ou níveis totais de tau, assim como estabilização de sua ligação aos microtúbulos poderiam ser benéficos ${ }^{21}$. Nos mamíferos, já foram identificadas mais de 20 proteínas responsáveis pela fosforilação da proteína tau. Dentre as fosfoquinases as 3 principais correspondem a CDK5, GSK3B e MARK ${ }^{22}$.

A GSK3 $\beta$ (Glicogênio Sintase Kinase 3-beta) foi até agora a mais utilizada como alvo terapêutico. Tentou-se a utilização de lítio ou ácido valproico como inibidores da GSK3 $\beta$ em indivíduos com PSP contudo os pacientes não toleraram a medicação ${ }^{8,21}$. Outro inibidor de GSK3 $\beta$ 
conhecido como tideglusib é inócuo e pode ser tentado em fases iniciais da doença e há estudos evidenciando menor atrofia em análise volumétrica, através de ressonância magnética ${ }^{21}$.

Houve também falha na tentativa de utilização de um estabilizador de microtúbulos, o davunetide ${ }^{8}$. Este é um composto formado por 8 aminoácidos representando epítopos da proteína neuroprotetora atividade dependente produzido pelas células gliais. Sua atividade seria através da manutenção do citoesqueleto neuronal por atuação nos microtúbulos 23 .

\section{O Azul De Metileno}

O azul de metileno (AzM) é uma droga anterior a fundação da FDA (Food and Drug Association) e aprovada em algumas condições clínicas como a metahemoglobinemia24. É usado na metahemoglobinemia, nas formas congênitas, ocupacionais e medicamentosas da doença. Atua transferindo elétrons para a metahemoglobina de forma não enzimática, restaurando a função da hemoglobina9 .

O AzM é também usado no tratamento da encefalopatia induzida por quimioterápicos como ifosfamida². A ifosfamida é um agente alquilante utilizado no tratamento de tumores sólidos, como sarcomas e em neoplasias hematológicas. Em 5 a $30 \%$ dos casos acarreta uma encefalopatia, cujo tratamento pode ser feito com AzM, de forma aguda ou profilática 9 . 
No choque séptico o AzM é usado na inibição das enzimas óxido nítrico sintase e da forma solúvel da guanilato ciclase responsáveis pelo processo de vasodilatação durante quadros de sepse ${ }^{12}$. O AzM pode ser utilizado no alívio do desconforto do trato urinário e em cirurgias como marcador de fístulas urinárias e gastrointestinais ${ }^{25}$. Também pode ser utilizado no tratamento do envenenamento por cianeto, um inibidor da cadeia respiratória mitocondrial ${ }^{24}$.

O AzM apresenta boa penetrabilidade através da barreira hematoencefálica com a da dose oral alcançando o cérebro rapidamente. Como pertence à classe das fenotiazinas, foi utilizada, por muito tempo, como molde para a descoberta de novas drogas desta classe ${ }^{26}$. Ele é adquirido como solução a $1 \%(10 \mathrm{~g} / \mathrm{l})$ e geralmente é administrado por via oral ou intravenosa, entretanto menos comumente pode ser administrado através de via intraóssea. Quando administrado por via oral, atinge a corrente sanguínea dentro de 1 a 2 horas ${ }^{10,27}$. A dose oral recomendada é de 50 a 300mg com dose terapêutica na faixa de 1 a $2 \mathrm{mg} / \mathrm{Kg} /$ dia e sua meia vida gira em torno de 10h. A Doença de Alzheimer é a tauopatia com maior número de pesquisas utilizando o medicamento, em doses habituais de $60 \mathrm{mg} 3 x /$ dia. Níveis tóxicos são esperados apenas em doses acima de 7,5mg/kg/dia, com formação de Corpos de Heinz nos eritrócitos ${ }^{12,25}$.

Os Corpos de Heinz representam agregados de hemoglobina desnaturada próximo a membrana celular dos glóbulos vermelhos. Modificam o formato das hemácias 
favorecendo sua hemólise assim como eliminação pela circulação esplênica ${ }^{28}$. Há contraindicação ao AzM na deficiência de glicose- 6 fosfato desidrogenase (G6PDH) ${ }^{29}$. A deficiência de G6PDH representa a mais frequente enfermidade por defeito enzimático do homem. Esta deficiência diminui a formação de glutationa reduzida, com dano oxidativo nas hemácias e episódios agudos de anemia hemolítica, sendo o AzM um fármaco capaz de acarretar uma crise hemolítica em portadores de G6PDH ${ }^{30}$.

Ademais, por atuar como um inibidor da monoamino oxidase $A(M A O-A)$, o AzM é contraindicado para pacientes sob uso de antidepressivos inibidores seletivos da recaptação de serotonina (ISRS) ${ }^{3}$.

Por último, mesmo nas doses clínicas habituais existe a possibilidade de alteração da coloração da urina. Isto também dificulta a sua utilização em pesquisas com placebo. Apesar destes problemas, é considerada droga segura, com relatos de crianças com níveis da droga superiores a 16 vezes a dose habitual. Nestes casos não houve paraefeitos relevantes, exceto coloração azulada da epiderme com reversão após a sua suspensão ${ }^{10}$.

Muitas questões relacionadas a ausência de pesquisas sobre o fármaco, especialmente em doenças parasitárias como a malária, devido ao seu baixo custo e pequeno interesse industrial farmacêutico ${ }^{12}$. 


\section{Ações do Azul de Metileno na agregação da proteína}

\section{Tau}

Devido as vantagens da utilização de drogas já disponíveis no mercado quando em comparação ao desenvolvimento de novas drogas, o AzM se apresenta como uma droga promissora pela sua segurança, baixo custo e capacidade de inibir a agregação da proteína tau 4 .

O AzM pode atuar como inibidor da fosforilação da proteica pela inibição da atividade da fosfoquinase MARK, diminuindo o desequilíbrio entre as formas fosforilada e não fosforilada da proteína tau ${ }^{22}$. Outro mecanismo de atuação do AzM nas tauopatias, seria diminuindo as formas solúveis de tau, mas não as formas agregadas da proteína ${ }^{24}$. Existe a possibilidade de as formas solúveis e oligoméricas de tau serem mais neurotóxicas quando comparadas a forma tau hiperfosforilada ${ }^{31}$.

O AzM pode afetar a fibrilação das formas $4 R$ da proteína tau de forma mais eficaz quando comparado as formas $3 R^{32}$. Pacientes com DA apresentam concentrações similares das formas $4 R$ e $3 R$ tau, enquanto pacientes com Paralisia Supranuclear Progressiva (PSP) predomínio das formas 4R sobre 3R tau ${ }^{5,16}$. Também a Degeneração Corticobasal (DCB) pode apresentar predomínio da forma 4R $\operatorname{tau}^{33}$.

Assim sendo, existe a possibilidade do AzM acarretar um efeito na agregação tau superior na PSP e na DCB quando comparado ao já mencionado e mais estudado efeito na DA. Diferentemente dos filamentos helicoidais pareados dos ENF 
da DA, os ENF de tau no PSP e DCB, formam agregados geralmente contendo filamentos retos ou contínuos ${ }^{34}$.

Por outro lado, na Demência Frontotemporal (DFT), há casos de predomínio tanto das formas 3R tau, como na variante de Pick e outros de predomínio $4 \mathrm{R}$ tau. Nestes os agregados tau levam a formação de emaranhados com filamentos em mola ou espiralados ${ }^{34}$. Embora esperado uma pior resposta das tauopatias com predomínio das formas $3 \mathrm{R}$ tau ao tratamento com AzM, existem relatos de indivíduos com DFT e melhora do quadro clínico após administração de doses orais de $A z M$ equivalentes as utilizadas nas pesquisas em $D^{35}$.

Um mecanismo alternativo da ação do AzM nas tauopatias seria a oxidação de resíduos de cisteína da proteína tau, causando sua manutenção na forma monomérica. Tal fato impediria a toxicidade neuronal pela formação de fibrilas e outros precursores tóxicos ${ }^{36}$.

A inibição da agregação poderia também ser decorrente do aumento da degradação lisossomal e autofagia dos componentes tau ${ }^{32}$. O cérebro durante a senescência diminui a atividade proteossomal neuronal, com depósito de agregados proteicos e outras proteínas mal enoveladas, dentre estas a proteína tau ${ }^{25}$. Este mecanismo justificaria os quadros de DA precoce ou familiar. Neste caso mutações de genes como da proteína precursora amiloide (PPA) ou da presenilina existentes na DA familiar ou precoce acarretam sobrecarga do sistema lisossomal. Esta sobrecarga pelo agregado de outras proteínas mal enoveladas além da 
proteína tau provocaria morte neuronal em idades mais jovens quando em comparação aos quadros típicos de DA ${ }^{37}$. Além disso, precursores da proteína tau como seus oligômeros e fibrilas podem ser transmitidos dos neurônios através de exocitose para uma célula vizinha, propagando seus efeitos tóxicos e assim ajudando na progressão da doença20.

O AzM poderia de forma alternativa estimular a autofagia da tau, através de outros mecanismos como a supressão de uma proteína celular chamada de mTOR (mammalian target of rapamicyn) ${ }^{38}$. Esta proteína corresponde a uma proteína kinase participante da homeostase de material proteico celular, facilitando a translação e inibindo a autofagia. Sua ativação ocasionaria aumento nos níveis de tau hiperfosforilada. Níveis de mTOR encontram-se aumentados no cérebro de indivíduos com DA, sendo esta via uma das implicadas no controle do envelhecimento celular ${ }^{39}$.

Outro mecanismo possível de ação do AzM seria através de proteínas chaperonas (proteínas participantes da montagem e construção de outras proteínas) responsáveis pela regulação e conformação da proteína tau. Neste caso o AzM atuaria inibindo a ação de proteínas de choque térmico chaperonas como a Hsp70ATPase, com consequente diminuição dos níveis de tau ${ }^{40}$. As chaperonas da família HSP70 estão entre as mais conservadas nas espécies animais e podem representar mais de $3 \%$ do total da massa 
proteica intracelular produzida de forma constitutiva mesmo fora de períodos de estresse térmico ${ }^{41}$.

Em contrapartida, não podemos descartar a hipótese do AzM atuar em doenças neurodegenerativas por mecanismos distintos de sua atuação na agregação tau. Considerando o fato do cérebro ser particularmente sensível a disfunção mitocondrial por estresse oxidativo ocasionado por sua alta taxa metabólica, o AzM é o primeiro composto químico capaz de induzir o complexo da cadeia respiratória mitocondrial. O AzM pode diminuir a produção de espécies reativas de oxigênio por atuação através de ativação do Complexo IV da cadeia respiratória (citocromo $\mathrm{C}$ oxidase) ${ }^{25}$.

Há a possibilidade de ser o efeito inibidor do AzM na agregação do $\beta$-amiloide, a sua verdadeira ação benéfica na DA, diferentemente das demais tauopatias ${ }^{24}$. Uma das possíveis explicações seria o fato de ser o $\beta$-amiloide um deflagrador da atividade neurotóxica da proteína tau nos ENF. Sendo assim o controle dos níveis de $\beta$-amiloide pelo AzM e não sua ação direta na agregação tau corresponderia ao seu verdadeiro benefício na DA. Tal efeito benéfico poderia não ocorrer em tauopatias sem a presença concomitante de material $\beta$-amiloide ${ }^{42,43}$.

Por último, não se encontrou na literatura, trabalhos utilizando o AzM na PSP apesar das evidências do uso do MB tauopatias. A maioria dos trabalhos tem como foco a Doença de Alzheimer pela sua frequência e impacto financeiro. Associado a isto, há raridade da PSP quando em comparação 
a DA, seu retardo diagnóstico e a inexistência de exame padrão ouro para diagnóstico.

\section{CONCLUSÃO}

Devido aos custos de produção de novos medicamentos é importante a utilização de substâncias já existentes no mercado em enfermidades distintas da inicial. Diversas doenças neurodegenerativas estão associadas a formação de agregados de proteínas. O Azul de Metileno apresenta baixo custo, boa tolerabilidade e uso já aprovado em outras condições clínicas. O efeito do Azul de Metileno na inibição de agregados de proteína tau já observado na Doença de Alzheimer e a Demência Frontotemporal, pode servir de modelo para sua utilização nas tautopatias, como a Paralisia Supranuclear Progressiva.

\section{REFERÊNCIAS}

1.Kaat DL, Chiu WZ, Boon AJW, van Swieten JC. Recent advances in progressive supranuclear palsy: a review. Curr Alzheimer Res 2011;8:295-302. https://doi.org/10.2174/156720511795563809

2. Owolabi L. Progressive supranuclear palsy misdiagnosed as Parkinson's disease: a case report and review of literature. Ann Med Health Sci Res.2013;3(Suppl 1):S44-7.

https://doi.org/10.4103/2141-9248.121221

3. Rampello L, Buttà V, Raffaele R, Vecchio I, Battaglia G, Cormaci G, et al. Progressive supranuclear palsy: A systematic review. Neurobiol Dis 2005;20:179-86. https://doi.org/10.1016/j.nbd.2005.03.013 4. Keith-Rokosh J, Ang LC. Progressive supranuclear palsy: a review of co-existing neurodegeneration. Can J Neurol Sci 2008;35:602-8. https://doi.org/10.1017/s0317167100009392

5.Long L, Cai X, Wei X, Liao JC, Xu YQ, Gao HM, et al. Progressive Supranuclear Palsy: What Do We Know About it? Curr Med Chem 2015;22:1182-93. 
https://doi.org/10.2174/0929867322666150302170552

6.Barsottini OGP, Felício AC, Aquino $\mathrm{CCH}$, Pedroso JL. Progressive supranuclear palsy: new concepts. Arq Neuropsiquiatr 2010;68:93846. https://doi.org/10.1590/s0004-282x2010000600020

7.Daniele A, Moro E, Bentivoglio AR. Zolpidem in Progressive Supranuclear Palsy. N Engl J Med 1999;341:543-4. https://doi.org/10.1056/NEJM199908123410721

8. Golbe LI. The tau of PSP: A long road to treatment. Mov Disord 2014;29:431-4. https://doi.org/10.1002/mds.25855

$9 . \mathrm{Oz} \mathrm{M}$, Lorke DE, Hasan M, Petroianu GA. Cellular and Molecular Actiones of Methylene Blue in the Nervous System. Med Res Rev 2012;31:93-117. https://doi.org/10.1002/med.20177

10.Oz M, Lorke DE, Petroianu GA. Methylene blue and Alzheimer's disease. Biochem Pharmacol 2009;78:927-32. https://doi.org/10.1016/j.bcp.2009.04.034

11.Paban V, Manrique C, Filali M, Maunoir-Regimbal S, Fauvelle F, Alescio-Lautier $B$. Therapeutic and preventive effects of methylene blue on Alzheimer's disease pathology in a transgenic mouse model. Neuropharmacology 2014;76:68-79.

https://doi.org/10.1016/j.neuropharm.2013.06.033

12.Schirmer $\mathrm{RH}$, Adler $\mathrm{H}$, Pickhardt M, Mandelkow E. "Lest we forget you - methylene blue...". Neurobiol Aging 2011;32:2325.e7-16. https://doi.org/10.1016/j. neurobiolaging.2010.12.012

13. Williams DR. Tauopathies: classification and clinical update on neurodegenerative diseases associated with microtubule-associated protein tau. Intern Med J 2006;36:652-60.

https://doi.org/10.1111/j.1445-5994.2006.01153.x

14.Clavaguera F, Lavenir I, Falcon B, Frank S, Goedert M, Tolnay M. "Prion-like" templated misfolding in tauopathies. Brain Pathol 2013;23:342-9. https://doi.org/10.1111/bpa.12044

15.Borroni B, Benussi A, Gazzini S, Turrone R , Gardoni F, Di Luca M, et al. Diagnosing Progressive Supranuclear Palsy: Role of Biological and Neuroimaging Markers. J Alzheimers Dis Park 2014;04:1-8. https://doi.org/10.4172/2161-0460.1000168

16.Luk C, Vandrovcova J, Malzer E, Lees A, de Silva R. Brain Tau Isoform mRNA and Protein Correlation in PSP Brain. Transl Neurosci 2010;1:30-6. https://doi.org/10.2478/v10134-010-0009-8

17.Williams DR, Lees AJ. Progressive supranuclear palsy: clinicopathological concepts and diagnostic challenges. Lancet Neurol 2009;8:270-9. https://doi.org/10.1016/S1474-4422(09)70042-0

18.Lee VM-Y, Goedert M, Trojanowski JQ. Neurodegenerative tauopathies. Annu Rev Neurosci 2001;24:1121-59. https://doi.org/10.1146/annurev.neuro.24.1.1121

19. Hattori M, Sugino E, Minoura K, In Y, Sumida M, Taniguchi T, et al. Different inhibitory response of cyanidin and methylene blue for filament formation of tau microtubule-binding domain. Biochem Biophys Res Commun 2008;374:158-63. https://doi.org/10.1016/j.bbrc.2008.07.001

20. Wischik CM, Harrington CR, Storey JMD. Tau-aggregation inhibitor 
therapy for Alzheimer's disease. Biochem Pharmacol 2014;88:529-39. https://doi.org/10.1016/j.bcp.2013.12.008

21.Stamelou M, Boxer AL. Disease-Modifying Treatments for Progressive Supranuclear Palsy. Mov Disord Clin Pract 2015;2:3-5. https://doi.org/10.1002/mdc3.12142

22.Sun W, Lee S, Huang X, Liu S, Inayathullah M, Kim K-M, et al. Attenuation of synaptic toxicity and MARK4/PAR1-mediated Tau phosphorylation by methylene blue for Alzheimer's disease treatment. Sci Rep 2016;6:34784. https://doi.org/10.1038/srep34784

23. Gold M, Lorenzl S, Stewart AJ, Morimoto BH, Williams DR, Gozes I. Critical appraisal of the role of davunetide in the treatment of progressive supranuclear palsy. Neuropsychiatr Dis Treat 2012;8:8593. https://doi.org/10.2147/NDT.S12518

24.Zakaria A, Hamdi N, Abdel-Kader RM. Methylene Blue Improves Brain Mitochondrial ABAD Functions and Decreases $A \beta$ in a Neuroinflammatory Alzheimer's Disease Mouse Model. Mol Neurobiol 2016;53:1220-8. https://doi.org/10.1007/s12035-014-9088-8

25. Atamna $H$, Kumar $R$. Protective role of methylene blue in Alzheimer's disease via mitochondria and cytochrome c oxidase. J Alzheimers Dis 2010;20:439-52. https://doi.org/10.3233/JAD-2010100414

26.van der Ven AT, Pape JC, Hermann D, Schloesser R, Genius J, Fischer $\mathrm{N}$, et al. Methylene Blue (Tetramethylthionine Chloride) Influences the Mobility of Adult Neural Stem Cells: A Potentially Novel Therapeutic Mechanism of a Therapeutic Approach in the Treatment of Alzheimer's Disease. J Alzheimers Dis 2017;57:531-40. https://doi.org/10.3233/JAD-160755

27.Sontag EM, Lotz GP, Agrawal N, Tran A, Aron R, Yang G, et al. Methylene Blue Modulates Huntingtin Aggregation Intermediates and Is Protective in Huntington's Disease Models. J Neurosci 2012; 32:11109-19. https://doi.org/10.1523/JNEUROSCI.0895-12.2012

28.Basile F, Di Santi A, Caldora M, Ferretti L, Bentivegna F, Pica A. Inclusion bodies in loggerhead erythrocytes are associated with unstable hemoglobin and resemble human Heinz bodies. J Exp Zool A Ecol Genet Physiol 2011;315:416-23. https://doi.org/10.1002/jez.687 29.Atamna H, Nguyen A, Schultz C, Boyle K, Newberry J, Kato H, et al. Methylene blue delays cellular senescence and enhances key mitochondrial biochemical pathways. FASEB J 2008;22:703-12. https://doi.org/10.1096/fj.07-9610com

30.Youngster I, Arcavi L, Schechmaster R, Akayzen Y, Popliski $\mathrm{H}$, Shimonov J, et al. Medications and glucose-6-phosphate dehydrogenase deficiency: an evidence-based review. Drug Saf 2010;33:713-26. https://doi.org/10.2165/11536520-00000000000000

31.Spires-Jones TL, Friedman T, Pitstick R, Polydoro M, Roe A, Carlson $\mathrm{GA}$, et al. Methylene blue does not reverse existing neurofibrillary tangle pathology in the rTg4510 mouse model of tauopathy. Neurosci Lett 2014;562:63-8. https://doi.org/10.1016/j. neulet.2014.01.013

32. Crowe A, James MJ, Lee VM, Smith AB 3rd, Trojanowski 
JQ, Ballatore C, et al. Aminothienopyridazines and methylene blue affect Tau fibrillization via cysteine oxidation. J Biol Chem 2013;288:11024-37. https://doi.org/10.1074/jbc.M112.436006

33.Kouri N, Whitwell JL, Josephs KA, Rademakers R, Dickson DW. Corticobasal degeneration: a pathologically distinct 4R tauopathy. Nat Rev Neurol 2011;7:263-72.

https://doi.org/10.1038/nrneurol.2011.43

34. Yoshida M. Cellular tau pathology and immunohistochemical study of tau isoforms in sporadic tauopathies. Neuropathology 2006;26:45770. https://doi.org/10.1111/j.1440-1789.2006.00743.x

35. Adler G, Mautes AE. Improvement in behavioral symptoms, motor impairment and activities of daily living in a patient with the behavioral variant of frontotemporal dementia under treatment with methylene blue. Geriatr Ment Heal Care 2014;2:1-2. https://doi.org/10.1016/j.gmhc. 2014.06.001

36.Pakavathkumar P, Sharma G, Kaushal V, Foveau B, LeBlanc AC. Methylene Blue Inhibits Caspases by Oxidation of the Catalytic Cysteine. Sci Rep 2015;5:13730. https://doi.org/10.1038/srep13730 37.Guzmán-Martinez L, Farías GA, Maccioni RB. Tau Oligomers as Potential Targets for Alzheimer's Diagnosis and Novel Drugs. Front Neurol 2013;4:167. https://doi.org/10.3389/fneur.2013.00167

38. Congdon EE, Wu JW, Myeku N, Figueroa YH, Herman M, Marinec PS, et al. Methylthioninium chloride (methylene blue) induces autophagy and attenuates tauopathy in vitro and in vivo. Autophagy 2012;8:60922. https://doi.org/10.4161/auto.19048

39. Caccamo A, Magrì A, Medina DX, Wisely EV, López-Aranda MF, Silva $\mathrm{AJ}$, et al. MTOR regulates tau phosphorylation and degradation: implications for Alzheimer's disease and other tauopathies. Aging Cell 2013;12:370-80. https://doi.org/10.1111/acel.12057

40.Jinwal UK, Miyata Y, Koren J 3rd, Jones JR, Trotter JH, Chang L, et al. Chemical manipulation of hsp70 ATPase activity regulates tau stability. J Neurosci 2009;29:12079-88.

https://doi.org/10.1523/JNEUROSCI.3345-09.2009

41.Finka A, Sharma SK, Goloubinoff P. Multi-layered molecular mechanisms of polypeptide holding, unfolding and disaggregation by HSP70/HSP110 chaperones. Front Mol Biosci 2015;2:29. https://doi.org/10.3389/fmolb.2015.00029

42.King ME, Kan H-M, Baas PW, Erisir A, Glabe CG, Bloom GS. Taudependent microtubule disassembly initiated by prefibrillar $\beta$-amyloid. J Cell Biol 2006;175:541-6. https://doi.org/10.1083/jcb.200605187 43. Rapoport M, Dawson HN, Binder LI, Vitek MP, Ferreira A. Tau is essential to $\beta$-amyloid-induced neurotoxicity. Proc Natl Acad Sci USA 2002;99:6364-9. https://doi.org/10.1073/pnas.092136199 